\title{
Custos variáveis de produção de Diachasmimorpha longicaudata (Ashmead) para controle de moscas-das-frutas
}

Variable costs of production for Diachasmimorpha longicaudata to control the fruit flies

\author{
Maria Gisely Camargos ${ }^{*}$, Maria de Lourdes Zamboni Costa ${ }^{2}$, Elisângela de Souza \\ Miranda ${ }^{3}$
}

\footnotetext{
1 Centro de Energia Nuclear na Agricultura, Universidade de São Paulo - Doutora em Ciências - Av. Centenário, 303, Caixa Postal 96, CEP 13400-970 - Piracicaba (SP), Brasil

2 Centro de Energia Nuclear na Agricultura, Universidade de São Paulo - Especialista em Manejo Integrado de Pragas e Receituário Agronômico - Laboratório de Radioentomologia - Av. Centenário, 303, Caixa Postal 96, CEP 13400-970 - Piracicaba (SP), Brasil

${ }^{3}$ Escola Superior de Agricultura "Luiz de Queiroz", Universidade de São Paulo - Doutora em Ciências (Microbiologia Agrícola) - Av. Pádua Dias, 11, Cx. Postal 9, CEP 13418-900 - Piracicaba (SP), Brasil
}

\section{Resumo}

O controle biológico aplicado consiste em liberações em massa de predadores ou parasitoides após a criação laboratorial em larga escala. Avaliar o custo de produção do parasitoide Diachasmimorpha longicaudata para controle biológico de moscas-das-frutas irá fornecer uma ferramenta capaz de auxiliar o planejamento, controle e uma forma de apoiar as empresas quanto as suas tomadas de decisão. Este trabalho teve por objetivo identificar e analisar os custos variáveis de produção do parasitoide $D$. longicaudata, criado em larvas de Anastrepha fraterculus utilizando a ferramenta de Custeio Baseado em Atividade [ABC]. Foi acompanhado o processo produtivo para obter o custo variável total de produção em laboratório de pesquisa e biofábrica de inimigos naturais, localizado em Piracicaba, São Paulo. A capacidade de produção de pupas de $A$. fraterculus parasitadas por $D$. longicaudata é de um milhão por semana nesse laboratório, sendo que um milhão de pupas representa $34 \mathrm{~L}$, e um $\mathrm{mL}$ contém aproximadamente 30 pupas. $\mathrm{O}$ custo variável de produção para produzir um milhão de pupas parasitadas por semana foi de $R \$ 5.919,65$ ou $R \$ 0,0059$ por pupa. O custo com mão de obra representa $57 \%$ do custo total, enquanto o custo com materiais representa $43 \%$. A tomada de decisão do produtor em relação ao controle biológico utilizando $D$. longicaudata dependerá de estudos prévios sobre o parasitoide e cultura pretendida.

Palavras-chave: controle biológico, custeio $\mathrm{ABC}$, produção de inimigos naturais

\begin{abstract}
Applied biological control consists in the mass liberation of laboratory reared predators or parasitoids. Evaluating the variable cost of production of the fruit flies parasitoid Diachasmimorpha longicaudata for biological control will provide a tool to assist the planning, control and a way to support businesses as their decision-making. This study aims to identify and analyze the variable costs production of $D$. longicaudata, created in larvas of Anastrepha fraterculus using the Activity-Based Costing Tool [ABC]. The total variable cost was obtained by means of monitoring the rearing in a biofactory located in Piracicaba, Sao Paulo. The pupa production capacity of $A$. fraterculus parasitized by $D$. longicaudata is a million a week in this lab being a million pupas around $34 \mathrm{~L}$ or 30 per $\mathrm{mL}$. The total cost of production to produce one million parasitized pupa per week was $\mathrm{R} \$ 5,919.65$ or $\mathrm{R} \$ 0.0059$ per pupa. The cost of labor is $57 \%$ of the total cost, while the cost of materials is $43 \%$. The producer's decision-making in relation to biological control using $D$. longicaudata depends on previous studies of the parasitoid and desired crop.
\end{abstract}

Keywords: Production of natural enemies, ABC expenditure, biological control

\footnotetext{
*Autor correspondente: <mariagisely@hotmail.com>

Enviado: 08 nov. 2016

Aprovado: 08 maio 2017
} 


\section{Introdução}

O Brasil é o terceiro maior produtor mundial de frutas com 42 milhões de toneladas produzidas de um total de 340 milhões de toneladas colhidas em todo 0 mundo por ano (Fachinello e Nachtigal, 2009). Em 2014 foram US\$ 636,402 milhões em exportações, representando apenas 3\% do que o país produz, e 3,21\% a menos do que em 2013 (Reetz et al., 2015). No entanto, a produtividade frutícola poderia ser maior se o controle de pragas fosse mais efetivo e disseminado.

As moscas-das-frutas (Diptera: Tephritide) são as pragas de maior expressão econômica na fruticultura mundial devido aos danos que causam aos frutos, aos elevados custos de controle e aos prejuízos com as restrições fitossanitárias impostas nas relações comerciais internacionais (Godoy et al., 2011). Os prejuízos ao livre comércio mundial de frutas motivados por pragas podem ultrapassar os $U \$ 2$ bilhões. No Brasil, esse valor situa-se entre U\$120 e 200 milhões anuais, oriundos das perdas diretas, custo de controle e perdas de mercados de exportação (Zucchi et al., 2004). Tradicionalmente, no país o controle de moscas-das-frutas, em nível de propriedade, é baseado principalmente na utilização de inseticidas na parte aérea da planta, nas formas de cobertura total ou de isca tóxica. Entretanto, a melhor estratégia a ser utilizada para o controle químico de moscas-das-frutas depende da espécie de mosca, da cultura explorada e da região a ser abrangida (Raga e Sato, 2016). O método de controle químico, embora produza resultados satisfatórios, apresentam alguns inconvenientes, como desequilíbrios ambientais, danos à saúde humana, ser contrário à opinião pública e adicionar resíduos aos produtos (Carvalho et al., 2000). Nesse sentido, o controle biológico é uma das mais promissoras alternativas ao uso de pesticidas no manejo de pragas.

No mundo todo, o controle biológico de moscas-das-frutas é baseado em liberações de parasitoides, produzidos em grande escala (criação massal) e liberados para agirem sobre a praga-alvo. O parasitoide Diachasmimorpha longicaudata (Ashmed) (Hymenoptera: Braconidae) é uma das espécies mais utilizadas devido à facilidade de criação massal e fácil adaptação às várias espécies de moscas-dasfrutas de importância econômica (Garcia e Ricalde, 2012; Ovruski et al., 2000). Para serem realizadas liberações inundativas desse parasitoide, duas criações são necessárias: a da mosca hospedeira e a do agente ativo.

Os custos elevados para implantação das biofábricas de produção massal e a baixa qualidade dos insetos produzidos são as principais limitações do controle biológico aplicado (Carvalho e Nascimento, 2002). Dessa forma, técnicas de criação 
que resultem em baixo custo de produção e em parasitoides de alta qualidade são sempre importantes e devem ser estimuladas (Sivinski et al., 1996).

Nesse sentido, a contabilidade de custos pode fornecer subsídios importantes para a execução de um projeto de criação massal de parasitoides. Uma vez que a contabilidade de custos tem duas funções relevantes: o auxílio ao controle e a ajuda às tomadas de decisões. No que diz respeito ao controle, sua mais importante missão é fornecer dados para o estabelecimento de padrões, orçamentos e outras formas de previsão e, num estágio imediatamente seguinte, acompanhar o efetivamente acontecido para comparação com os valores anteriormente definidos (Martins, 2010).

A determinação de custos, em um determinado período, é definida de acordo com o volume de produção. Assim, os custos variáveis podem apresentar mudanças em seus valores conforme sua produção. Em relação aos custos fixos, não ocorre variação de acordo com o volume produzido (Ferreira, 2007).

Um dos sistemas de custos é o Custeio Baseado em Atividade conhecido como Activity-Based Costing $[A B C]$. É um sistema de análise de custos que tem como objetivo reduzir as distorções provocadas pelo rateio dos custos indiretos de fabricação (Martins, 2010). Segundo Faria e Costa (2010), o método de custeio baseado em atividades busca identificar as atividades relacionadas a um produto ou serviço, rastreando os recursos consumidos por estas atividades, utilizando-se de vários direcionadores de custo.

Como o controle dos gastos com a produção e a lucratividade são fatores de relevância para as organizações, nada melhor do que além de ter o parasitoide disponível para atender a demanda do mercado, que se tenha um controle funcional de formação de custos. Portanto, avaliar o custo de produção do D. longicaudata para controle de moscas-das-frutas, fornecerá uma ferramenta capaz de auxiliar o planejamento, controle e uma forma de apoiar as empresas quanto as suas tomadas de decisão. Dessa forma, o trabalho teve por objetivo identificar e analisar os custos variáveis de produção do parasitoide $D$. longicaudata, criado em larvas de Anastrepha fraterculus, utilizado no controle biológico das moscas-das-frutas.

\section{Material e Métodos}

Esta pesquisa foi realizada em laboratório de pesquisa e biofábrica de inimigos naturais localizado em Piracicaba, São Paulo. A criação de insetos foi realizada em salas climatizadas ( $26 \pm 2^{\circ} \mathrm{C} ; 70-80 \%$ de UR e fotofase de $14 \mathrm{~h}$ ). Nesse laboratório as metodologias de criação em laboratório foram testadas e permitiram 0 
desenvolvimento da tecnologia necessária para a produção desses insetos em grande escala no Brasil.

Para a análise do custo de produção do parasitoide $D$. longicaudata foi necessário acompanhar o processo produtivo para obter o custo operacional de produção. Para melhor desenvolvimento, conforme enfatizado neste estudo, foi utilizado o Sistema de Custeio ABC (Martins, 2010; Faria e Costa, 2010).

\section{Processo produtivo de Diachasmimorpha longicaudata}

A biofábrica contou com um espaço com cerca de $300 \mathrm{~m}^{2}$, dividido em salas para criação de insetos, controle de qualidade, preparo de dietas e lavagem de material. A metodologia de criação foi adaptada do método usado na Division of Plant Industry [DPI] de Gainesville, Flórida, EUA (Burns, 1993). Esse laboratório foi pioneiro em multiplicar massalmente este parasitoide em larvas de C. capitata com sucesso (Walder e Sarriés, 1995). O parasitoide foi multiplicado em larvas de A. fraterculus (Figura 1) e a criação em grande escala de $A$. fraterculus foi estabelecida com sucesso após o período de domesticação da espécie, obtendo-se larvas de boa qualidade.

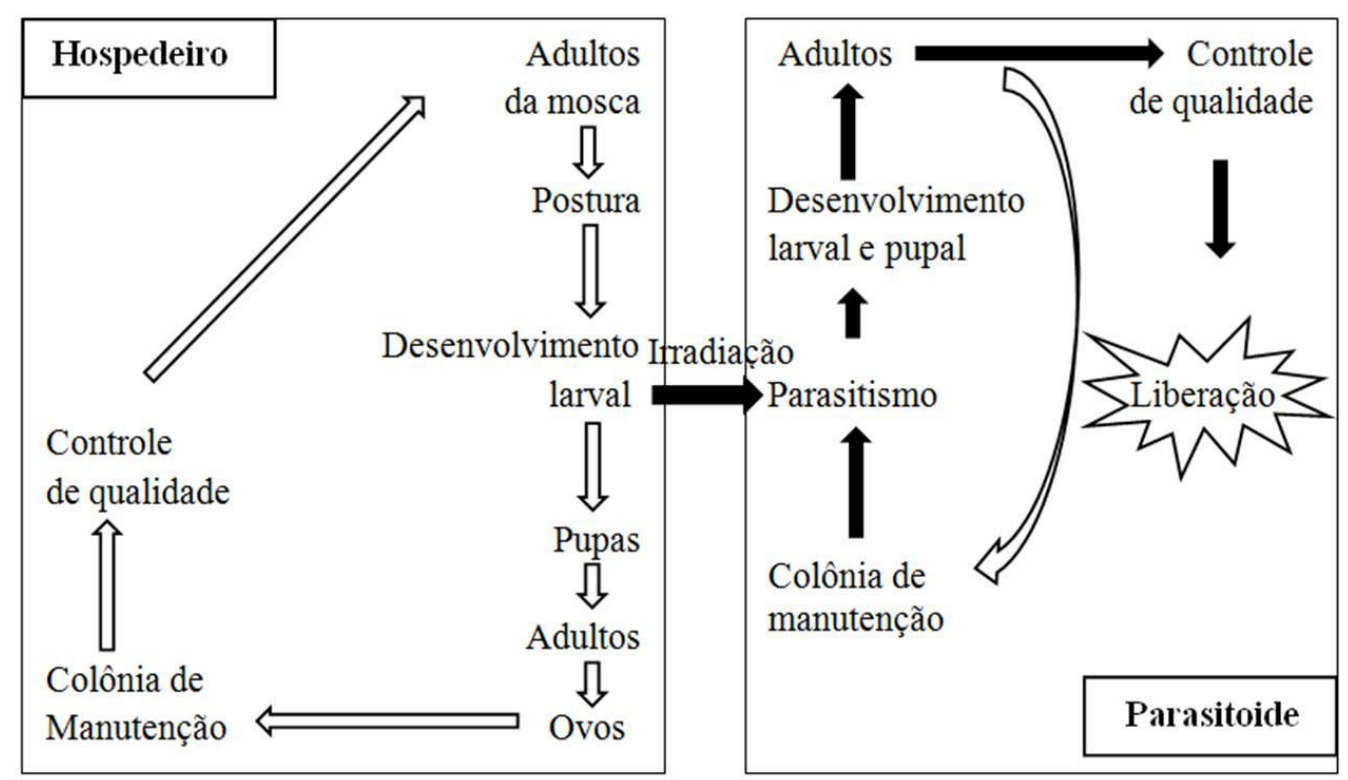

Figura 1. Esquema de criação de Diachasmimorpha longicaudata em larvas Irradiadas de Anastrepha fraterculus

Fonte: Adaptado de Parra et al. (2002) 
O uso de larvas irradiadas de $A$. fraterculus para a produção de $D$. longicaudata promoveu maior emergência do parasitoide, com maior quantidade e melhor qualidade de fêmeas que larvas de Ceratitis capitata'1.

\section{Criação artificial do hospedeiro Anastrepha fraterculus}

A metodologia de domesticação e criação artificial de $A$. fraterculus foi descrita por Morelli-de-Andrade ${ }^{2}$.

\section{Controle de qualidade e dados de produção em laboratório}

Durante a criação da mosca em condições artificiais, foram realizadas amostragens para o acompanhamento da qualidade do inseto produzido. Os parâmetros adotados foram fornecidos pela Seção de Entomologia da Agência Internacional de Energia Nuclear (IAEA, 2003).

Toda coleta de ovos, larvas e pupas foram volumetricamente medidas e amostras de ovos e pupas foram retiradas para avaliação biológica. Para cada lote produzido, separaram amostras e este procedimento se repetiu a cada coleta por geração ${ }^{3}$.

\section{Criação de Diachasmimorpha longicaudata}

Em 1994, o laboratório recebeu os primeiros exemplares do parasitoide D. longicaudata, recém-importado, provenientes do Laboratório Embrapa Meio Ambiente, em Jaguariúna, SP (Walder e Sarriés, 1995). O Laboratório de Irradiação de Alimentos e Radioentomologia [LIARE] do Centro de Energia Nuclear na Agricultura [CENA] da Universidade de São Paulo passou a produzir massalmente esse parasitoide desde então (Walder e Sarriés, 1995).

Os exemplares de $D$. longicaudata foram criados utilizando-se larvas de $3^{\circ}$ estádio de $A$. fraterculus como hospedeiro. Esses parasitoides foram mantidos no Laboratório de Criação em condições controladas (temperatura de $26 \pm 2{ }^{\circ} \mathrm{C}$, UR de $65 \pm 10 \%$ e fotofase de $14 \mathrm{~h}$ ). Os adultos do parasitoide foram mantidos em gaiolas (50 x 50 x $30 \mathrm{~cm}$ ) com arestas de alumínio, duas laterais e o fundo fixo de acrílico, duas

\footnotetext{
1 Morelli-de-Andrade, R. 2013. Produção em grande escala do parasitoide Diachasmimorpha longicaudata (Hymenoptera: Braconidae) em larvas hospedeiras de Anastrepha fraterculus e Ceratitis capitata (Diptera: Tephritidae) linhagem mutante ts/-Viena. Tese de Doutorado em Ciências. Escola Superior de Agricultura Luiz de Queiroz, Universidade de São Paulo, Piracicaba, São Paulo, Brasil.

2 Morelli-de-Andrade. Ibid.

${ }^{3}$ Camargos, M.G. 2010. Moscas frugívoras (Díptera: Tephritoidea) em cafezais irrigados no norte de Minas Gerais: diversidade e controle biológico. Dissertação de Mestrado em Produção Vegetal no Semiárido. Universidade Estadual de Montes Claros, Janaúba, Minas Gerais,Brasil.
} 
laterais e o topo removíveis de tela, com livre acesso a uma "massa" feita de lenço de papel picado e mel para alimentação. As gaiolas foram dispostas em estantes de alumínio com rodas, de forma a ficarem três gaiolas sobrepostas com espaço suficiente entre elas para colocar as unidades de parasitismo e para a entrada de luz.

Larvas nuas (sem dieta) de $A$. fraterculus, no fim do terceiro ínstar (irradiadas com 40 Gy) foram expostas ao parasitoide em unidades de parasitismo [UPs], as quais consistiram em anéis de tubo de PVC de $10 \mathrm{~cm}$ de diâmetro com $1 \mathrm{~cm}$ de altura.

O fundo da UP foi confeccionado em tecido voil colado no anel, e a tampa removível e feita de PVC. Cada UP acomodou de 20 a $25 \mathrm{~mL}$ de larvas, correspondendo acerca de 600-800 indivíduos. As larvas foram acondicionadas, as unidades tampadas e colocadas externamente na parte superior das gaiolas de adultos do parasitoide, onde ficaram por $50 \mathrm{~min}$ à disposição das fêmeas para o parasitismo. Da mesma forma, foi também montada uma UP com larvas não irradiadas para o controle de qualidade.

\section{Controle de qualidade permanente}

De cada lote de parasitismo, foram retiradas amostras de 100 pupários irradiados e não-irradiados para controle de qualidade avaliando-se os seguintes parâmetros:

- Taxa de parasitismo: determinada pela amostra de larvas não-irradiadas parasitadas, calculada pela eq.(1).

$$
\text { Taxa de parasitismo }=\frac{n^{0} \text { de parasitoides emergidos }}{\left(n^{2} \text { de parasitoides }+n^{2} \text { de moscas }\right)}
$$

- Emergência do parasitoide, calculada pela eq.(2).

$$
\text { Emergência do parasitoide }=\frac{\mathrm{n}^{\circ} \text { de parasitoides }}{\mathrm{n}^{\circ} \text { total de pupários }}
$$

- Razão sexual, calculada pela eq.(3).

$$
\text { Razão sexual }=\frac{\mathrm{n}^{\circ} \text { de fêmeas }}{\mathrm{n}^{0} \text { machos }+\mathrm{n}^{\circ} \text { fềmeas }}
$$

Os parâmetros acima descritos foram avaliados rotineiramente, em todos os lotes de parasitismo, entretanto outros parâmetros de qualidade foram avaliados periodicamente, tais como: 
- Habilidade de voo: 100 pupas parasitadas (com 24 h antes da emergência). A habilidade de voo foi expressa como taxa de voadores, calculada pela seguinte eq.(4).

$$
\text { Número de pupas }=\frac{\text { pupas não emergidas }+ \text { semi }- \text { emergidas }+ \text { não }- \text { voadores }}{n^{0} \text { de pupas }}
$$

- Longevidade sob estresse: confinou-se 10 machos e 10 fêmeas, separadamente, em potes plásticos transparentes de $500 \mathrm{~mL}$, sem alimento e água. Após a emergência dos adultos, iniciou-se a contagem diária da mortalidade;

- Tamanho e peso dos parasitoides: o tamanho dos parasitoides, da cabeça à extremidade do abdômen, e do ovipositor das fêmeas, foram medidos em estereomicroscópio com ocular micrométrica. O peso foi determinado utilizando balança semi-analítica.

O modelo Activity-Based Costing para laboratório de criação massal de

\section{Diachasmimorpha longicaudata}

As informações contábeis úteis para o gerenciamento foram extraídas por meio de sistemas de custos denominados custeios. Os custeios consistem em apropriar todos os custos e despesas geradas para a produção dos bens e dos serviços. Os custeios foram responsáveis por mensurar a quantidade proporcional de custos relevante a cada unidade de produto/serviço (Martins, 2010).

Para estimar com maior precisão os custos das atividades laboratoriais foi aplicado o método de custeio $A B C$, pois este se mostrou o mais adequado para as situações com maior diversidade de tipos de serviços. Segundo Martins (2010), uma vez que os processos foram compostos por atividades que se inter-relacionam, tal análise permite uma visualização das atividades que podem ser melhoradas, reestruturadas ou até mesmo eliminadas, reduzindo os custos de um determinado processo produtivo, de forma a melhorar o desempenho competitivo da empresa.

O sistema $\mathrm{ABC}$ tem como fundamento básico a busca do princípio da causação, ou seja, identificar, por meio de rastreamento, o agente causador do custo, para the imputar o valor. A idéia básica foi atribuir primeiramente os custos às atividades e posteriormente atribuir custos das atividades aos produtos. Sendo assim, primeiramente fez-se o rastreamento dos custos que cada atividade causou, atribuindo-Ihes estes custos, e posteriormente verificaram como os portadores finais 
de custos consumiram serviços das atividades, atribuindo-lhes os custos definidos (Martins, 2003).

Nesse sentido, a escolha dos direcionadores foi o grande diferencial. Segundo Martins (2010), o direcionador de custos foi o fator que determina o custo de uma atividade. Para efeito de custeio de produtos, o direcionador deve ser o fator que determina ou influencia a maneira de como os produtos "consomem" as atividades.

Desta forma, o direcionador de custos foi à base utilizada neste trabalho para atribuir os custos das atividades aos parasitoides produzidos. Primeiramente foram identificados os custos das atividades e em sequência os custos das atividades aos parasitoides produzidos.

\section{Resultados e Discussão}

\section{Coleta de dados financeiros}

Inicialmente, diversos materiais foram identificados para compor os custos diretos, os quais foram custos de produtos usados diretamente na realização das atividades, tais como bandejas, gaiolas de criação de insetos, etc. Entretanto, para o levantamento dos custos de produção de $D$. longicaudata foram considerados apenas os custos variáveis operacionais. Justifica-se tal utilização, pois trata-se de laboratório de pesquisa que se encontrava em operação, portanto o custo operacional foi obtido por meio de informações e análises realizadas no laboratório durante o mês de março do ano de 2013. Sendo assim, os custos fixos não foram considerados no presente trabalho.

A capacidade de produção de pupas de $A$. fraterculus parasitadas por D. longicaudata é de um milhão por semana no laboratório, sendo que um milhão de pupas representa $34 \mathrm{~L}$ e $1 \mathrm{~mL}$ contém aproximadamente 30 pupas. No laboratório, foram utilizadas seis pessoas, sendo dois técnicos trabalhando oito horas por dia e quatro estagiários trabalhando quatro horas por dia, durante cinco dias da semana, para a produção de um milhão de parasitoides por semana. Os cálculos para a mão de obra dos técnicos foram feitos considerando o seu salário bruto mensal acrescido dos encargos e benefícios trabalhistas que têm direito. O cálculo da mão de obra dos estagiários foi considerado somente o valor bruto da bolsa concedida a cada estagiário. 


\section{Análise dos Resultados}

Nesse trabalho, utilizou-se o Método de Custeio $A B C$, onde foram identificadas as etapas do processo de produção de $D$. longicaudata para controle de moscas-dasfrutas, alocando-as em departamentos formando assim o custo de cada um, o estudo levou em consideração apenas os custos de mão de obra e materiais.

O custo de mão de obra por departamento foi calculado por meio da quantificação do tempo que cada colaborador dedica em determinado departamento, obtendo-se, assim, o custo total de mão de obra igual a $\mathrm{R} \$ 3.355,25$ por semana (Tabela 1), para a produção de um milhão de pupas parasitadas por $D$. longicaudata. O departamento de Controle de Qualidade foi o que demandou maior utilização de mão de obra, que está relacionado ao tempo dedicado pelos técnicos a este departamento.

A qualificação da mão de obra foi muito importante para o desenvolvimento do processo produtivo, portanto destaca-se a importância da remuneração adequada aos colaboradores para que se alcance sucesso no resultado final do processo, que, neste trabalho, foram as pupas parasitadas por $D$. longicaudata. O produto final com qualidade irá apresentar maior eficiência no campo, ou seja, maior controle de moscas-das-frutas em pomares e plantações e isso representarão maior satisfação do consumidor, nesse caso o agricultor, e também a redução dos seus custos de produção, além da redução da utilização de produtos químicos no controle de pragas.

Tabela 1. Custo de mão de obra por departamento para a produção de um milhão de parasitoides por semana com dois técnicos trabalhando oito horas por dia, e quatro estagiários trabalhando quatro horas por dia, durante cinco dias da semana

\begin{tabular}{|c|c|c|c|}
\hline Departamento & Custo por semana & Custo por mês & Custo por ano \\
\hline & & -- $R \$$------. & \\
\hline Criação do hospedeiro & 838,81 & $3.355,25$ & $40.262,95$ \\
\hline Criação do parasitoide & 503,29 & $2.013,15$ & $24.157,77$ \\
\hline Dieta (alimentação) & 335,52 & $1.342,10$ & $16.105,18$ \\
\hline Irradiação & 167,76 & 671,05 & $8.052,59$ \\
\hline Controle de qualidade & $1.174,34$ & $4.697,34$ & $56.368,12$ \\
\hline Lavanderia & 335,52 & $1.342,10$ & $16.105,18$ \\
\hline Total & $3.355,25$ & $13.420,98$ & $161.051,78$ \\
\hline
\end{tabular}

Fonte: Resultados originais da pesquisa

Os materiais e respectivos custos utilizados no processo de produção de larvas de $A$. fraterculus parasitadas com $D$. longicaudata estão descritos na Tabela 2. $O$ custo com materiais foi de $R \$ 2.564,40$ por semana para produção de um milhão de 
pupas parasitadas por $D$. longicaudata. $O$ material que representa o maior custo foi o ágar, devido ao seu custo unitário ser elevado (Figura 2).

Tabela 2. Custo dos materiais para produção de pupas parasitadas por Diachasmimorpha longicaudata

\begin{tabular}{|c|c|c|c|c|c|c|c|}
\hline \multirow{2}{*}{ Insumo } & \multirow{2}{*}{ Unidade } & \multirow{2}{*}{ Preço } & \multicolumn{2}{|c|}{ Quantidade } & \multicolumn{3}{|c|}{ Custo } \\
\hline & & & Semana & Mês & Semana & Mês & Ano \\
\hline & & $--R \$--$ & & & & $R \$-$ & \\
\hline Açúcar cristal & $\mathrm{kg}$ & 2,12 & 32,71 & 130,83 & 69,34 & 277,36 & 3328,32 \\
\hline Levedura & $\mathrm{kg}$ & 4,73 & 32,71 & 130,83 & 154,70 & 618,81 & 7425,70 \\
\hline Germe de Trigo & $\mathrm{t}$ & 25,00 & 39,72 & 158,86 & 817,66 & $3.270,66$ & 39247,86 \\
\hline Agar & $\mathrm{kg}$ & 180,00 & 5,45 & 21,80 & 981,20 & $3.924,79$ & 47097,44 \\
\hline Nipagin & $\mathrm{kg}$ & 44,00 & 0,44 & 1,74 & 19,19 & 76,75 & 921,02 \\
\hline Ácido clorídrico & $\mathrm{kg}$ & 18,00 & 3,27 & 13,08 & 58,87 & 235,49 & 2825,85 \\
\hline Benzoato de sódio & $\mathrm{kg}$ & 12,80 & 0,55 & 2,18 & 6,98 & 27,91 & 334,92 \\
\hline Biones & $\mathrm{kg}$ & 25,80 & 7,01 & 28,03 & 180,82 & 723,28 & 8679,38 \\
\hline Açúcar Refinado & $\mathrm{kg}$ & 2,69 & 21,03 & 84,10 & 56,56 & 226,24 & 2714,83 \\
\hline Álcool & $\mathrm{L}$ & 12,00 & 4,36 & 17,44 & 52,33 & 209,32 & 2511,86 \\
\hline Hipoclorito de Sódio & $\mathrm{L}$ & 0,68 & 5,00 & 20,00 & 3,40 & 13,60 & 163,20 \\
\hline Algodão (Pçt 25g) & $\mathrm{Pc}$ & 1,23 & 1,00 & 4,00 & 1,23 & 4,92 & 59,04 \\
\hline Detergente & $\mathrm{L}^{3}$ & 1,00 & 3,00 & 12,00 & 3,00 & 12,00 & 144,00 \\
\hline Papel Higiênico & $\mathrm{RI}$ & 0,92 & 3,00 & 12,00 & 2,76 & 11,04 & 132,48 \\
\hline Esponja (Pçt3ud) & $\mathrm{Pc}$ & 10,00 & 15,00 & 60,00 & 150,00 & 600,00 & 7200,00 \\
\hline Mel de abelha & $\mathrm{L}$ & 30,00 & 0,21 & 0,85 & 6,36 & 25,46 & 305,20 \\
\hline Total & & & & & $2.564,40$ & 10257,63 & 123091,10 \\
\hline
\end{tabular}

Fonte: Resultados originais da pesquisa

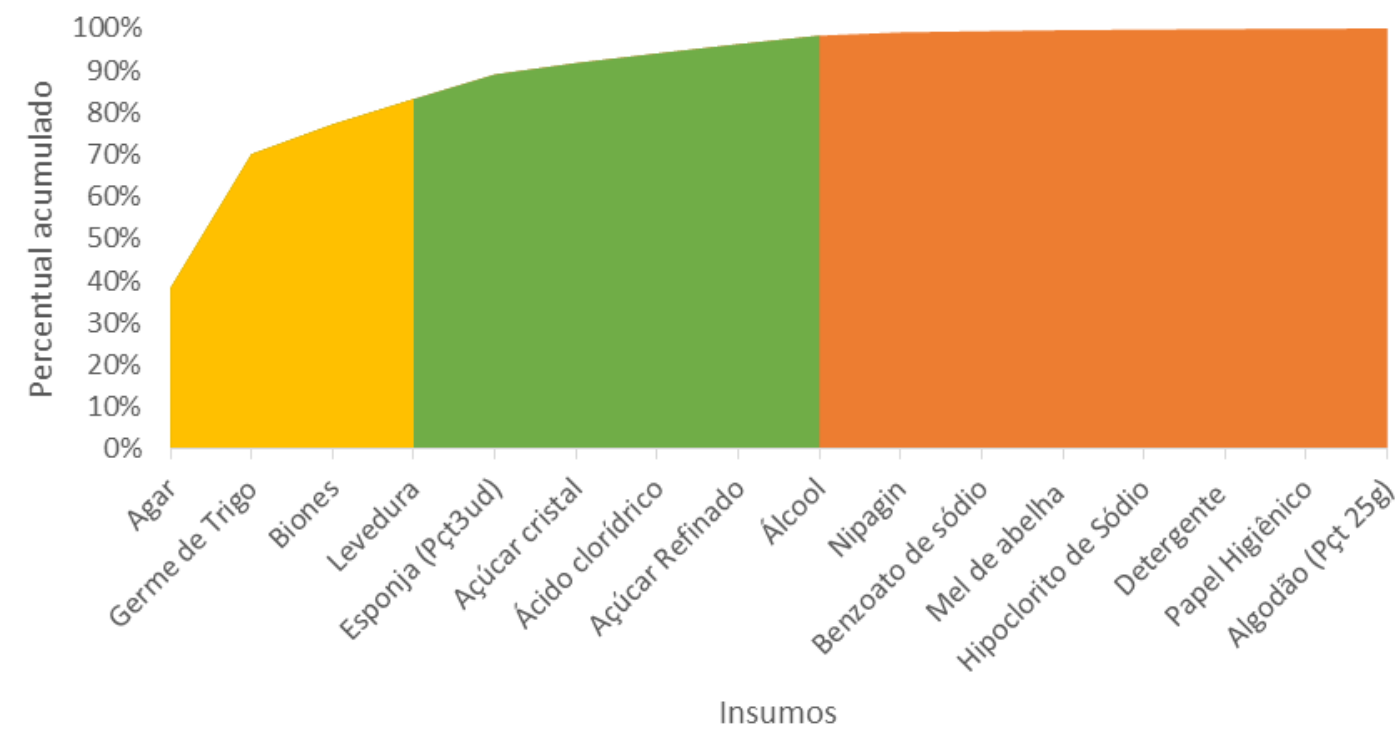

Figura 2. Activity-Based Costing $[A B C]$ para os insumos utilizados na produção de pupas parasitadas por Diachasmimorpha longicaudata

Fonte: Resultados originais da pesquisa 
O custo operacional total de produção para produzir um milhão de pupas parasitadas por D. longicaudata por semana foi de $\mathrm{R} \$ 5.919,65$ (Tabela 3). O custo com mão de obra representou $57 \%$ do custo total, enquanto o custo com materiais representou 43\%. Observou a importância da mão de obra na formação do custo total, sendo que foi necessária a participação de dois técnicos no processo de produção de pupas parasitadas por $D$. longicaudata.

Tabela 3. Custo total de produção de Diachasmimorpha longicaudata, por semana, mês e ano

\begin{tabular}{|c|c|c|c|c|}
\hline \multirow{2}{*}{$\begin{array}{l}\text { Materiais por } \\
\text { semana }\end{array}$} & \multirow{2}{*}{$\begin{array}{c}\text { Mão de obra por } \\
\text { semana }\end{array}$} & \multicolumn{3}{|c|}{ Total } \\
\hline & & Semana & Mês & Ano \\
\hline & & $--R \$$ & & \\
\hline $2.564,40$ & $3.355,25$ & $5.919,65$ & $23.678,58$ & $284.142,98$ \\
\hline
\end{tabular}

Fonte: Resultados originais da pesquisa

A curva $A B C$ é um método de classificação de dados para se separar os itens de maior importância que frequentemente apresentam em menor número (Carvalho, 2002). Na Figura 2 pode-se observar que quatro insumos (ágar, germe de trigo, biones e levedura) correspondem por mais de $80 \%$ dos custos com materiais, e, portanto, os insumos restantes representaram menos de $20 \%$ dos custos com materiais, de forma que um possível aumento no preço de um desses quatro insumos irá afetar em maior proporção o aumento no custo de produção de pupas parasitadas por D. longicaudata, uma vez que esses insumos representaram a maior parte do custo de produção.

Consequentemente, qualquer esforço para reduzir o consumo nesses insumos também reduzirá o custo de produção de $D$. longicaudata.

Deste modo, a curva ABC possibilitou a análise de sensibilidade, demonstrando a concentração de esforços na redução dos custos da produção onde o impacto foi maior. Uma redução de $10 \%$ nos custos dos quatro insumos mais custosos impactaria em uma redução de $8 \%$ dos custos dos insumos. Enquanto uma redução dos mesmos $10 \%$ nos custos de todos os outros insumos representaria uma redução de apenas $2 \%$.

Morelli-de-Andrade ${ }^{4}$ testou a redução dos $10 \mathrm{~g}$ iniciais de ágar que eram usados na dieta original de Salles (1992), para 3,4 g e percebeu que a redução proporcionou uma melhora no peso das pupas. Segundo o autor, o uso de

\footnotetext{
${ }^{4}$ Cf. nota 1
} 
ingredientes nacionais na composição das dietas e o baixo consumo de ágar podem reduzir os custos de produção. Desse modo, pequenas modificações podem ser feitas para melhorar o rendimento do processo de produção. Ceteris paribus, essa redução de $66 \%$ no uso do mais custoso insumo acarretaria em redução de $21,36 \%$ no custo total dos insumos ou redução de $9,25 \%$ do custo incluindo a mão de obra.

O custo para produção de um milhão de pupas por semana foi de $\mathrm{R} \$ 5.919,65$, portanto, o custo de uma pupa parasitada por $D$. longicaudata foi $R \$ 0,0059$ considerando um milhão de pupas por semana no laboratório, $34 \mathrm{~L}$ de pupas e aproximadamente 30 pupas por $\mathrm{mL}$. Entretanto, esses valores acompanham a inflação e dessa forma, os custos poderão variar conforme os valores dos produtos no mercado.

É muito difícil separar os problemas de uma criação do inseto hospedeiro daqueles ligados à produção do parasitoide (Parra et al., 2002). No caso de $D$. longicaudata, trata-se, no caso da criação da mosca hospedeira $A$. fraterculus e do inimigo natural, da criação de duas espécies, e esse foi o grande fator complicador. Há necessidade, portanto, de conhecer a biologia, ecologia, comportamento, fisiologia, nutrição etc. da mosca hospedeira e do parasitoide e, evidentemente, suas interrelações para a implantação da criação massal.

Criações massais envolveram produções diárias de muitos insetos e dessa forma, houve necessidade de contratar funcionários capacitados ou realizar treinamentos para a criação dos insetos. Nesse caso, além dos problemas biológicos de criação, surgem outros, como inventário, compra e armazenamento de material e manutenção das instalações e equipamentos. À medida que aumentou o número de insetos produzidos, cresceram os problemas relacionados a instalações, custos, microrganismos (contaminantes) e controle de qualidade dos insetos (Parra et al., 2002). Essa criação envolveu diferentes tecnologias que utilizaram dietas naturais e artificiais e condições de ambiente controladas (Cano et al., 2006). O laboratório foi a base física e técnica de produção do parasitoide e, para isso, deve possuir uma infraestrutura com equipamentos, instalações, pessoal e dimensionamento adequado.

O conhecimento prévio da capacidade de parasitismo e dispersão de $D$. longicaudata foi uma ferramenta importante na determinação do número de pontos de liberação e, consequentemente, na elaboração de metodologias que viabilizaram a eficiência deste parasitoide no campo, principalmente em liberações inundativas ${ }^{5}$. Dessa forma, a determinação do número de pontos de liberação por unidade de área

\footnotetext{
${ }^{5}$ Cf. nota 3
} 
possui papel fundamental na adoção do programa de liberação de um agente de controle biológico, uma vez que os custos de liberação foram diretamente relacionados a este número (Zachrisson e Parra, 1998).

As características intrínsecas de cada cultura, em função da condição microclimática criada pela mesma, podem afetar essa capacidade de busca, pela variação da temperatura em cada condição (Biever, 1972). Diferentes distâncias do local de liberação podem resultar em variabilidade na taxa de parasitismo em uma dada cultura, como consequência das características biológicas do parasitoide, por exemplo, a sua capacidade de voo e/ou as características inerentes à própria cultura o que pode funcionar como uma barreira física e dificultando a dispersão dos parasitoides. Portanto, estas variações dependem tanto do tipo e do desenvolvimento da cultura estudada quanto das características biológicas do parasitoide escolhido (Bueno et al., 2012).

Os padrões de dispersão de $D$. longicaudata em citros no interior de São Paulo foram avaliados por Paranhos et al. (2007), e verificaram que os parasitoides chegaram a uma distância média de $40 \mathrm{~m}$, dispersando-se mais durante o verão do que no inverno. Em liberação de $D$. longicaudata realizada em pomar de goiaba na região norte do Estado do Rio de Janeiro, Leal et al. (2008) observaram a visitação de fêmeas o parasitoide em unidades de parasitismo dispostas a $20 \mathrm{~m}$ de distância do ponto de liberação, após $24 \mathrm{~h}$ da liberação do parasitoide.

A dispersão de $D$. longicaudata criado no hospedeiro $C$. capitata em cafezal irrigado foi avaliada por Camargos $(2010)^{6}$ durante os meses de dezembro a abril na região norte de Minas Gerais, região de clima considerado semi-árido com temperaturas médias de 25ำ C. A distância média percorrida pelo parasitoide, $24 \mathrm{~h}$ após a liberação, foi de 35,2 m e a área de dispersão média de 1.899,3 $\mathrm{m}^{2}$. Esse mesmo autor recomendou que fossem liberados 12.000 parasitoides distribuídos em seis pontos por hectare na cultura do café. Portanto, o custo de controle biológico com o parasitoide $D$. longicaudata, estudado no presente trabalho, ficaria em $R \$ 70,80$ por hectare, na qual seriam necessários 12.000 parasitoides liberados em um hectare ao custo de $R \$ 0,0059$ cada parasitoide na cultura do café. Para o controle da mesma mosca, Ceratitis capitata, na cultura dos citros, um dos produtos registrados no Ministério da Agricultura, Pecuária e Abastecimento [MAPA] é um piretroide usado em forma de isca tóxica e de cobertura, com recomendação de uso de cerca de 300-360 $\mathrm{mL}$ do produto comercial por hectare (MAPA, 2017). Embora, neste caso, o custo de

\footnotetext{
${ }^{6}$ Cf. nota 3
} 
controle seja menor, deve ser observado que se trata de culturas diferentes. Além disto, as aplicações do produto necessitam de 28 dias de carência para evitar que permaneçam resíduos nos frutos. O que não ocorre quando são liberados inimigos naturais. Diversos outros produtos são autorizados pelo MAPA para controle de moscas-das-frutas (Raga e Sato, 2016). Com isso, observa-se a importância do cálculo do custo de produção dos parasitoides, porque assim, o agricultor poderá prever o impacto da utilização do método de controle biológico na cultura em questão.

O mercado de produção de parasitoides ainda é recente. Esse mercado representa menos de $2 \%$ dos produtos de proteção de cultivos, segundo estimativa do MAPA, que projeta aumento de participação do setor para $20 \%$ até 2020 (MAPA, 2015). O Brasil é um exemplo de sucesso no uso de biodefensivos, principalmente com produtos à base de Bacillus thuringiensis, Baculovírus, Metarhizium, Trichoderma e Cotesia, entre outros. Contudo ainda não há registros sobre o custo para produção de $D$. longicaudata, portanto, este estudo trata-se de estudo pioneiro e, nesse caso, não foi possível realizar uma análise de mercado.

Fica evidente a necessidade de novos estudos a respeito do custo de produção de parasitoides para controle biológico, pois é uma alternativa interessante para reduzir a utilização de produtos químicos e, certamente, uma alternativa mais econômica.

\section{Conclusão}

O trabalho permite identificar que o custo para a produção de um milhão de pupas parasitadas do parasitoide Diachasmimorpha longicaudata criado em larvas de Anastrepha fraterculus analisados através da ferramenta de Custeio Baseado em Atividade corresponde a $\mathrm{R} \$ 5.919,65$.

A partir dessa análise verifica-se que dentre os insumos utilizados na produção, os que são responsáveis por mais de $80 \%$ dos custos são o ágar, germe de trigo, biones e levedura. Portanto, um aumento no preço destes insumos resultará na elevação no custo de produção das pupas parasitadas por Diachasmimorpha longicaudata. 


\section{Referências}

Biever, K.D. 1972. Effect of the temperature on the rate of search by Trichogramma and its potential application in field releases. Environmental Entomology 1:194 -197

Bueno, R.C.O.F.; Parra, J.R.P.; Bueno, F.A. 2012. Trichogramma pretiosum parasitism and dispersal capacity: a basis for developing biological control programs for soybean caterpillars. Bulletin of Entomological Research 102(1): 1-8.

Burns, R.E. 1993. Caribbean fruit fly parasitóide rearing and release procedure Manual. Departament of Agriculture \& Consume Service, Gainesville, Florida, USA.

Cano, M.A.V; Santos, E.M.; Pinto, A.S. 2006. Produção de Cotesia flavipes para controle da broca-da-cana. p. 21-24. In: Pinto, A. de S. Controle de pragas da cana de açúcar. Biocontrol, Sertãozinho, Minas Gerais, Brasil.

Carvalho, J.M.C. de. 2002. Logística. 3ed. Edições Silabo, Lisboa, Portugal.

Carvalho, R.S.; Nascimento, A.S. 2002. Criação e utilização de Diachasmimorpha longicaudata para controle biológico de moscas-das-frutas (Tephritidae). p. 165-179. In: Parra, J.R.P.; Botelho, P.S.M.; Corrêa-Ferreira, B.S.; Bento, J.M.S. (Ed.). Controle biológico no Brasil: Parasitóides e Predadores, São Paulo, SP, Brasil.

Carvalho, R.S.; Nascimento; A.S.; Matrangolo, W.J.R. 2000. Controle biológico. Cap. 9, p. 113-117. In: Malavasi, A.; Zucchi, R.A. (Ed.). Moscas-das-frutas de importância econômica no Brasil: conhecimento básico e aplicado. Holos Editora, Ribeirão Preto, São Paulo, Brasil.

Fachinello, J.C.; Nachtigal, J.C. 2009. Fruticultura: fundamentos e práticas.

Disponível em:

<http://www.cpact.embrapa.br/publicacoes/download/livro/fruticultura_fundamentos_pr atica/1.1.htm>. Acesso em: 12 set.2015.

Faria, A.C.; Costa, M.F.G. 2010. Gestão de custos logísticos. Atlas, São Paulo, São Paulo, Brasil.

Ferreira, J.A. 2007. Contabilidade de custos. Editora Ferreira, Rio de Janeiro, Rio de Janeiro, Brasil.

Garcia, F.R.M., Ricalde, M.P. 2012. Augmentative biological control using parasitoids for fruit fly management in Brazil. Insects, Basel 4: 55-70.

Godoy, M.J.S; Pacheco, W.S.P., Malavasi, A. 2011. Moscas-das-frutas quarentenárias para o Brasil. p. 111. In: Silva, R.A., Lemos, W.P., Zucchi, R.A. Moscas-das-frutas na Amazônia brasileira: diversidade, hospedeiros e inimigos naturais. Embrapa. Macapá, Amapá, Brasil.

International Atomic Energy Agency. IAEA. 2003. Manual for product quality control and shipping procedures for sterile mass-reared tephritid fruit flies, Version 5.0.

International Atomic Energy Agency. Vienna, Austria. 85 pp. 
Leal, R.M.; Aguiar-Menezes, E.L.; Lima Filho, M.; Ribeiro, J.C.R.; Menezes, E.B. 2008. Capacidade de sobrevivência e dispersão de Diachasmimorpha longicaudata, um parasitoide exótico de larva de moscas-das-frutas. Embrapa Agrobiologia. Seropédica, Rio de Janeiro, Brasil.

Martins, E. 2003. Contabilidade de custos. 9ed. Atlas. São Paulo, São Paulo, Brasil.

Martins, E. 2010. Contabilidade de Custos. 10ed. Atlas. São Paulo, São Paulo, Brasil.

Ministério da Agricultura, Pecuária e Abastecimento [MAPA]. 2015. Projeções do agronegócio: Brasil 2014/15 a 2024/25, Projeções de Longo Prazo. Biblioteca Nacional de Agricultura - BINAGRI. Disponível em:

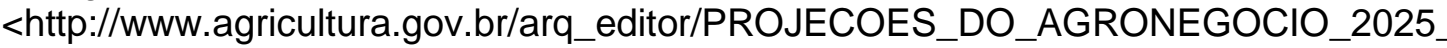
WEB.pdf>. Acesso em: 27 jan 2015.

Ministério da Agricultura, Pecuária e Abastecimento [MAPA]. 2017. Produtos Formulados. Disponível em:

$<$ http://agrofit.agricultura.gov.br/agrofit_cons/principal_agrofit_cons>. Acesso em: 15 fev. 2017.

Raga, A.; Sato, M.E. 2016. Controle químico de moscas-das-frutas. Disponível em: <http://www.biologico.agricultura.sp.gov.br/docs/dt/moscas_das_frutas.pdf $>$. Acesso em: 21 mar. 2017.

Reetz, E.R.; Kist, B.B.; Santos, C.E.; Carvalho, C.; Drum, M. 2015. Anuário brasileiro da fruticultura 2014. Editora Gazeta Santa Cruz, Santa Cruz do Sul, Rio Grande do Sul, Brasil.

Salles, L.A.B. 1992. Metodologia de criação de Anastrepha fraterculus (Wiedemann, 1830) (Diptera: Tephritidae) em dieta artificial em laboratório. Anais da Sociedade Entomológica do Brasil, 21: 479-486.

Sivinski, J.; Calkins, C.O.; Baranowski, R.; Harris, D.; Brambila, J.; Diaz, J.; Burns, R.E.; Holler, T.; Dodson, G. 1996. Suppression of a Caribbean fruit by (Anastrepha suspensa (Loew), Diptera: Tephiritidae) population through augmented releases of the parasitoid Diachasmimorpha longicaudata (Ashmead) (Hymenoptera: Braconidae). Biological Control 6: 177-185.

Ovruski, S.M.; Aluja, M.; Sivinski, J.; Wharton, R.A. 2000. Hymenopteran parasitoids on fruit-infesting Tephritidae (Diptera) in Latin America and the southern United State: diversity, distribution, taxonomic status and their use in fruit fly biological control. Integrated Pest Management Reviews 5: 81-107.

Paranhos, B.A.J.; Mendes, P.C.D.; Papadopoulos, N.T.; Walder, J.M.M. 2007. Dispersion patterns of Diachasmimorpha longicaudata (Hymenoptera: Braconidae) in Citrus orchards in southeast Brazil. Biocontrol Science and Technology 17: 375-385.

Parra, J.R.P.; Botelho, P.S.M.; Corrêa-Ferreira, B.S.; Bento, J.M.S. 2002. Controle biológico: Uma visão inter e multidisciplinar. p.125-137. In: Parra, J.R.P.; Botelho, P.S.M.; Corrêa-Ferreira, B.S.; Bento, J.M.S. (Ed.). Controle biológico no Brasil: parasitoides e predadores. Manole, São Paulo, Brasil. 
Walder, J.M.M., Sarriés, S.R.V. 1995. Introdução do parasitóide Diachasmimorpha longicaudata (Ashmead) (Hymenoptera: Braconidae) para o controle de moscas-dasfrutas no Estado de São Paulo. In: Resumos do 15을 Congresso Brasileiro de Entomologia, Caxambu.

Zachrisson, B.; Parra, J.R.P. 1998. Capacidade de dispersão de Trichogramma pretiosum Riley, 1879 para o controle de Anticarsia gemmatalis Hubner, 1818 em soja. Scientia Agricola 55(1).

Zucchi, R.A.; Malavasi, A., Nascimento, A.S.; Walder, J.M.M. 2004. Prejuízos das moscas-das-frutas na exportação de citros. Visão Agrícola 2: 72-77. 